International Electronic Journal of Geometry

Volume 7 No. 1 PP. 36-43 (2014) C)IEJG

\title{
SEMIPARALLEL TENSOR PRODUCT SURFACES IN $\mathbb{E}^{4}$
}

\author{
BETÜL BULCA AND KADRI ARSLAN \\ Dedicated to memory of Proffessor Franki Dillen
}

\begin{abstract}
B.Y. Chen initiated the study of the tensor product immersion of two immersions of a given Riemannian manifold. Inspired by Chen's definition, F. Decruyenaere, F. Dillen, L. Verstraelen and L. Vrancken studied the tensor product of two immersions, in general, different manifolds; under certain conditions, this realizes an immersion of the product manifold. Further, tensor product surfaces of Euclidean plane curves were investigated by I.Mihai and B. Rouxel. In the present study we consider the tensor product surfaces in 4-dimensional Euclidean space $\mathbb{E}^{4}$. We have shown that tensor product surfaces in $\mathbb{E}^{n}$ satisfying the semiparallelity condition $\bar{R}(X, Y) \cdot h=0$ are totally umbilical surfaces.
\end{abstract}

\section{INTRODUCTION}

Recall definitions and results of [3]. Let $M$ and $N$ be two differentiable manifolds and $f: M \rightarrow \mathbb{E}^{m}, h: N \rightarrow \mathbb{E}^{n}$ two immersions. The tensor product map $f \otimes h$ : $M \times N \rightarrow \mathbb{E}^{m n}$ is defined by $(f \otimes h)(p, q)=f(p) \otimes h(q)$. The necessary and sufficient conditions for $f \otimes h$ to be an immersion were obtained in [5] . Further, tensor products of spherical and equivariant immersions were studied in [4] by F. Decruyenaere, F. Dillen, L. Verstraelen and one of the present authors. For many immersions $f, h$ which are not transversal, the tensor product $f \otimes h$ is still worthwhile to be investigated and in many cases still produces an immersion. Tensor product surfaces of Euclidean plane curves were studied by the Ion Mihai and B. Rouxel [11] and tensor product surfaces of Lorentzian plane curves by Ion Mihai, I. Van de Woestyne, L. Verstraelen and J. Walrave [14] (see also [1]). As such, in the following sections, we will consider the tensor product immersions, actually surfaces in $\mathbb{E}^{4}$, which are obtained from a Euclidean space curve and a Euclidean plane curve.

Let $M$ a submanifold of a $(n+d)$-dimensional Euclidean space $\mathbb{E}^{n+d}$. Denote by $\bar{R}$ the curvature tensor of the Vander Waerden-Bortoletti connection $\bar{\nabla}$ of $M$ and $h$ is the second fundamental form of $M$ in $\mathbb{E}^{n+d}$. The submanifold $M$ is called semi-parallel (or semi-symmetric [10]) if $\bar{R} \cdot h=0$ [6]. This notion is an extrinsic analogue for semi-symmetric spaces, i.e. Riemannian manifolds for which $R \cdot R=0$ and a direct generalization of parallel submanifolds, i.e. submanifolds for which

2010 Mathematics Subject Classification. 53C15,53C40.

Key words and phrases. Normal curvature, Tensor product surface, Semiparallel surface. 
$\bar{\nabla} h=0$. In [6] J. Deprez showed the fact that the submanifold $M \subset \mathbb{E}^{n+d}$ is semiparallel implies that $(M, g)$ is semi-symmetric. For references on semi-symmetric spaces, see [16]; for references on parallel immersions, see [8]. In [6] J. Deprez gave a local classification of semi-parallel hypersurfaces in Euclidean space. It is easily seen that all surfaces are semi-parallel.

In the present study we consider the tensor product surfaces in 4-dimensional Euclidean space $\mathbb{E}^{4}$. We have shown that tensor product surfaces in $\mathbb{E}^{4}$ satisfying the semiparallelity condition $\bar{R}(X, Y) \cdot h=0$ are totally umbilical.

\section{BASIC CONCEPTS}

Let $M$ be a smooth surface in $\mathrm{n}$-dimensional Euclidean space $\mathbb{E}^{n}$ given with the surface patch $X(u, v):(u, v) \in D \subset \mathbb{E}^{2}$. The tangent space to $M$ at an arbitrary point $p=X(u, v)$ of $M$ span $\left\{X_{u}, X_{v}\right\}$. In the chart $(u, v)$ the coefficients of the first fundamental form of $M$ are given by

$$
E=\left\langle X_{u}, X_{u}\right\rangle, F=\left\langle X_{u}, X_{v}\right\rangle, G=\left\langle X_{v}, X_{v}\right\rangle,
$$

where $\langle$,$\rangle is the Euclidean inner product. We assume that W^{2}=E G-F^{2} \neq 0$, i.e. the surface patch $X(u, v)$ is regular. For each $p \in M$, consider the decomposition $T_{p} \mathbb{E}^{n}=T_{p} M \oplus T_{p}^{\perp} M$ where $T_{p}^{\perp} M$ is the orthogonal component of the tangent plane $T_{p} M$ in $\mathbb{E}^{n}$, that is the normal space of $M$ at $p$.

Let $\chi(M)$ and $\chi^{\perp}(M)$ be the space of the smooth vector fields tangent and normal to $M$ respectively. Denote by $\nabla$ and $\bar{\nabla}$ the Levi-Civita connections on $M$ and $\mathbb{E}^{n}$, respectively. Given any vector fields $X_{i}$ and $X_{j}$ tangent to $M$ consider the second fundamental map $h: \chi(M) \times \chi(M) \rightarrow \chi^{\perp}(M)$;

$$
h\left(X_{i}, X_{j}\right)=\widetilde{\nabla}_{X_{i}} X_{j}-\nabla_{X_{i}} X_{j} ; 1 \leq i, j \leq 2 .
$$

where $\widetilde{\nabla}$ is the induced. This map is well-defined, symmetric and bilinear.

For any normal vector field $N_{\alpha} 1 \leq \alpha \leq n-2$ of $M$, recall the shape operator $A: \chi^{\perp}(M) \times \chi(M) \rightarrow \chi(M)$

$$
A_{N_{\alpha}} X_{i}=-\widetilde{\nabla}_{N_{\alpha}} X_{i}+D_{X_{i}} N_{\alpha} ; \quad 1 \leq i \leq 2 .
$$

where $D$ denotes the normal connection of $M$ in $\mathbb{E}^{n}[2]$.This operator is bilinear, self-adjoint and satisfies the following equation:

$$
h_{i j}^{\alpha}=\left\langle A_{N_{\alpha}} X_{i}, X_{j}\right\rangle=\left\langle h\left(X_{i}, X_{j}\right), N_{\alpha}\right\rangle, 1 \leq i, j \leq 2,1 \leq \alpha \leq n-2 .
$$

The equation (2.2) is called Gaussian formula, and

$$
h\left(X_{i}, X_{j}\right)=\sum_{\alpha=1}^{n-2} h_{i j}^{\alpha} N_{\alpha}, \quad 1 \leq i, j \leq 2
$$

where $h_{i j}^{\alpha}$ are the coefficients of the second fundamental form $h$ [2]. If $h=0$ then $M$ is called totally geodesic. $M$ is totally umbilical if all shape operators are proportional to the identity map. $M$ is an isotropic surface if for each $p$ in $M$, $\|h(X, X)\|$ is independent of the choice of a unit vector $X$ in $T_{p} M$.

If we define a covariant differentiation $\bar{\nabla} h$ of the second fundamental form $h$ on the direct sum of the tangent bundle and normal bundle $T M \oplus T^{\perp} M$ of $M$ by

$$
\left(\bar{\nabla}_{X_{i}} h\right)\left(X_{j}, X_{k}\right)=D_{X_{i}} h\left(X_{j}, X_{k}\right)-h\left(\nabla_{X_{i}} X_{j}, X_{k}\right)-h\left(X_{j}, \nabla_{X_{i}} X_{k}\right)
$$


for any vector fields $X_{i}, X_{j}, X_{k}$ tangent to $M$. Then we have the Codazzi equation

$$
\left(\bar{\nabla}_{X_{i}} h\right)\left(X_{j}, X_{k}\right)=\left(\bar{\nabla}_{X_{j}} h\right)\left(X_{i}, X_{k}\right)
$$

where $\bar{\nabla}$ is called the Vander Waerden-Bortoletti connection of $M[2]$.

We denote $R$ and $\bar{R}$ the curvature tensors associated with $\nabla$ and $D$ respectively;

$$
\begin{aligned}
R\left(X_{i}, X_{j}\right) X_{k} & =\nabla_{X_{i}} \nabla_{X_{j}} X_{k}-\nabla_{X_{j}} \nabla_{X_{i}} X_{k}-\nabla_{\left[X_{i}, X_{j}\right]} X_{k}, \\
R^{\perp}\left(X_{i}, X_{j}\right) N_{\alpha} & =h\left(X_{i}, A_{N_{\alpha}} X_{j}\right)-h\left(X_{j}, A_{N_{\alpha}} X_{i}\right) .
\end{aligned}
$$

The equation of Gauss and Ricci are given respectively by

$(2.11)\left\langle R^{\perp}\left(X_{i}, X_{j}\right) N_{\alpha}, N_{\beta}\right\rangle=\left\langle\left[A_{N_{\alpha}}, A_{N_{\beta}}\right] X_{i,} X_{j}\right\rangle$

for the vector fields $X_{i}, X_{j}, X_{k}$ tangent to $M$ and $N_{\alpha}, N_{\beta}$ normal to $M$ [2].

Let us $X_{i} \wedge X_{j}$ denote the endomorphism $X_{k} \longrightarrow\left\langle X_{j}, X_{k}\right\rangle X_{i}-\left\langle X_{i}, X_{k}\right\rangle X_{j}$. Then the curvature tensor $R$ of $M$ is given by the equation

$$
R\left(X_{i}, X_{j}\right) X_{k}=\sum_{\alpha=1}^{n-2}\left(A_{N_{\alpha}} X_{i} \wedge A_{N_{\alpha}} X_{j}\right) X_{k} .
$$

It is easy to show that

$$
R\left(X_{i}, X_{j}\right) X_{k}=K\left(X_{i} \wedge X_{j}\right) X_{k} .
$$

where $K$ is the Gaussian curvature of $M$ defined by

$$
K=\left\langle h\left(X_{1}, X_{1}\right), h\left(X_{2}, X_{2}\right)\right\rangle-\left\|h\left(X_{1}, X_{2}\right)\right\|^{2}
$$

(see $[9])$.

The normal curvature $K_{N}$ of $M$ is defined by (see [5])

$$
K_{N}=\left\{\sum_{1=\alpha<\beta}^{n-2}\left\langle R^{\perp}\left(X_{1}, X_{2}\right) N_{\alpha}, N_{\beta}\right\rangle^{2}\right\}^{1 / 2} .
$$

We observe that the normal connection $D$ of $M$ is flat if and only if $K_{N}=0$, and by a result of Cartan, this equivalent to the diagonalisability of all shape operators $A_{N_{\alpha}}$ of $M$, which means that $M$ is a totally umbilical surface in $\mathbb{E}^{n}$.

Further, the mean curvature vector $\vec{H}$ of $M$ is defined by

$$
\vec{H}=\frac{1}{2} \sum_{\alpha=1}^{n-2} \operatorname{tr}\left(A_{N_{\alpha}}\right) N_{\alpha} \text {. }
$$

\section{SEmi-PARAllel Surfaces}

Let $M$ a smooth surface in $n$-dimensional Euclidean space $\mathbb{E}^{n}$. Let $\bar{\nabla}$ be the connection of Vander Waerden-Bortoletti of $M$. Denote the tensors $\bar{\nabla}$ by $\bar{R}$. Then the product tensor $\bar{R} \cdot h$ of the curvature tensor $\bar{R}$ with the second fundamental form $h$ is defined by

$$
\begin{aligned}
\left(\bar{R}\left(X_{i}, X_{j}\right) \cdot h\right)\left(X_{k}, X_{l}\right)= & \bar{\nabla}_{X_{i}}\left(\bar{\nabla}_{X_{j}} h\left(X_{k}, X_{l}\right)\right)-\bar{\nabla}_{X_{j}}\left(\bar{\nabla}_{X_{i}} h\left(X_{k}, X_{l}\right)\right) \\
& -\bar{\nabla}_{\left[X_{i}, X_{j}\right]} h\left(X_{k}, X_{l}\right)
\end{aligned}
$$

for all $X_{i}, X_{j}, X_{k}, X_{l}$ tangent to $M$. 
The surface $M$ is said to be semi-parallel if $\bar{R} \cdot h=0$, i.e. $\bar{R}\left(X_{i}, X_{j}\right) \cdot h=0$ ([10], $[6],[7],[15])$. It is easy to see that

$$
\begin{aligned}
\left.(3.1) \bar{R}\left(X_{i}, X_{j}\right) \cdot h\right)\left(X_{k}, X_{l}\right)= & R^{\perp}\left(X_{i}, X_{j}\right) h\left(X_{k}, X_{l}\right) \\
& -h\left(R\left(X_{i}, X_{j}\right) X_{k}, X_{l}\right)-h\left(X_{k}, R\left(X_{i}, X_{j}\right) X_{l}\right),
\end{aligned}
$$

This notion is an extrinsic analogue for semi-symmetric spaces, i.e. Riemannian manifolds for which $R \cdot R=0$ and a generalization of parallel surfaces, i.e. $\bar{\nabla} h=0$ [8].

First, we give the following well-known results.

Lemma 3.1. [6] Let $M \subset \mathbb{E}^{n}$ a smooth surface given with the patch $X(u, v)$. Then the following equalities are hold;

$$
\begin{aligned}
\left(\bar{R}\left(X_{1}, X_{2}\right) \cdot h\right)\left(X_{1}, X_{1}\right)= & \left(\sum_{\alpha=1}^{n-2} h_{11}^{\alpha}\left(h_{22}^{\alpha}-h_{11}^{\alpha}\right)+2 K\right) h\left(X_{1}, X_{2}\right) \\
& +\sum_{\alpha=1}^{n-2} h_{11}^{\alpha} h_{12}^{\alpha}\left(h\left(X_{1}, X_{1}\right)-h\left(X_{2}, X_{2}\right)\right) \\
(3.2)\left(\bar{R}\left(X_{1}, X_{2}\right) \cdot h\right)\left(X_{1}, X_{2}\right)= & \left(\sum_{\alpha=1}^{n-2} h_{12}^{\alpha}\left(h_{22}^{\alpha}-h_{11}^{\alpha}\right)\right) h\left(X_{1}, X_{2}\right) \\
& +\left(\sum_{\alpha=1}^{n-2} h_{12}^{\alpha} h_{12}^{\alpha}-K\right)\left(h\left(X_{1}, X_{1}\right)-h\left(X_{2}, X_{2}\right)\right) \\
\left(\bar{R}\left(X_{1}, X_{2}\right) \cdot h\right)\left(X_{2}, X_{2}\right)= & \left(\sum_{\alpha=1}^{n-2} h_{22}^{\alpha}\left(h_{22}^{\alpha}-h_{11}^{\alpha}\right)-2 K\right) h\left(X_{1}, X_{2}\right) \\
& +\sum_{\alpha=1}^{n-2} h_{22}^{\alpha} h_{12}^{\alpha}\left(h\left(X_{1}, X_{1}\right)-h\left(X_{2}, X_{2}\right)\right) .
\end{aligned}
$$

Proof. Substituting (2.5) and (2.4) into (2.9) we get

$$
\begin{aligned}
R^{\perp}\left(X_{1}, X_{2}\right) N_{\alpha}= & h_{12}^{\alpha}\left(h\left(X_{1}, X_{1}\right)-h\left(X_{2}, X_{2}\right)\right) \\
& +\left(h_{22}^{\alpha}-h_{11}^{\alpha}\right) h\left(X_{1}, X_{2}\right) .
\end{aligned}
$$

Further, by the use of (2.13) we get

$$
\begin{aligned}
& R\left(X_{1}, X_{2}\right) X_{1}=-K X_{2} \\
& R\left(X_{1}, X_{2}\right) X_{2}=K X_{1} .
\end{aligned}
$$

So, substituting (3.3) and (3.4) into (3.1) we get the result.

Semi-parallel surfaces in $\mathbb{E}^{n}$ are classified by J. Deprez [6]:

Theorem 3.1. [6] Let $M$ a surface in n-dimensional Euclidean space $\mathbb{E}^{n}$. Then $M$ is semi-parallel if and only if locally;

i) $M$ is equivalent to a 2-sphere, or

ii) $M$ has trivial normal connection, or

iii) $M$ is an isotropic surface in $\mathbb{E}^{5} \subset \mathbb{E}^{n}$ satisfying $\|H\|^{2}=3 K$. 


\section{Semi-Parallel Tensor Product Surfaces in $\mathbb{E}^{4}$}

In the following sections, we will consider the tensor product immersions, actually surfaces in $\mathbb{E}^{4}$, which are obtained from two Euclidean plane curves. We recall definitions and results of [5]. Let $c_{1}: \mathbb{R} \rightarrow \mathbb{E}^{2}$ and $c_{2}: \mathbb{R} \rightarrow \mathbb{E}^{2}$ be two Euclidean curves. Put $c_{1}(t)=(\gamma(t), \delta(t))$ and $c_{2}(s)=(\alpha(s), \beta(s))$. Then their tensor product surface is given by patch

$$
f=c_{1} \otimes c_{2}: \mathbb{R}^{2} \rightarrow \mathbb{E}^{4}
$$

$$
f(t, s)=(\alpha(s) \gamma(t), \beta(s) \gamma(t), \alpha(s) \delta(t), \beta(s) \delta(t)) .
$$

(see [13] and [4]). If we take $c_{1}$ as an unit plane circle centered at 0 and $c_{2}(s)=$ $(\alpha(s), \beta(s))$ is an Euclidean plane curve. Then the surface patch becomes

$$
M: \quad f(t, s)=(\alpha(s) \cos t, \beta(s) \cos t, \alpha(s) \sin t, \beta(s) \sin t) .
$$

An orthonormal tangent basis on $M$ is given by

$$
\begin{aligned}
& X_{1}=\frac{1}{\left\|c_{2}\right\|} \frac{\partial f}{\partial t}, \\
& X_{2}=\frac{1}{\left\|c_{2}^{\prime}\right\|} \frac{\partial f}{\partial s} .
\end{aligned}
$$

The normal space of $M$ is spanned by

$$
\begin{gathered}
N_{1}=\frac{1}{\left\|c_{2}^{\prime}\right\|}\left(-\beta^{\prime}(s) \cos t, \beta^{\prime}(s) \cot s, \alpha^{\prime}(s) \sin t,-\alpha^{\prime}(s) \sin t\right), \\
N_{2}=\frac{1}{\left\|c_{2}\right\|}(-\beta(s) \sin t, \beta(s) \sin t, \alpha(s) \cos t,-\alpha(s) \cos t) .
\end{gathered}
$$

By covariant differentiation with respect to $X_{1}$ and $X_{2}$ a straightforward calculation gives

$$
\begin{aligned}
\tilde{\nabla}_{X_{1}} X_{1} & =-a(s) X_{2}+b(s) N_{1}, \\
\tilde{\nabla}_{X_{2}} X_{2} & =c(s) N_{1}, \\
\tilde{\nabla}_{X_{2}} X_{1} & =b(s) N_{2}, \\
\tilde{\nabla}_{X_{1}} X_{2} & =a(s) X_{1}-b(s) N_{2},
\end{aligned}
$$

and

$$
\begin{aligned}
\tilde{\nabla}_{X_{1}} N_{1} & =-b(s) X_{1}-a(s) N_{2}, \\
\tilde{\nabla}_{X_{1}} N_{2} & =b(s) X_{2}+a(s) N_{1}, \\
\tilde{\nabla}_{X_{2}} N_{1} & =-c(s) X_{2}, \\
\tilde{\nabla}_{X_{2}} N_{2} & =-b(s) X_{1},
\end{aligned}
$$

where

$$
\begin{aligned}
& a(s)=\frac{\alpha(s) \alpha^{\prime}(s)+\beta(s) \beta^{\prime}(s)}{\left\|c_{2}(s)\right\|^{2}\left\|c_{2}^{\prime}\right\|}, \\
& b(s)=\frac{\alpha(s) \beta^{\prime}(s)-\beta(s) \alpha^{\prime}(s)}{\left\|c_{2}(s)\right\|^{2}\left\|c_{2}^{\prime}\right\|}, \\
& c(s)=\frac{\alpha^{\prime}(s) \beta^{\prime \prime}(s)-\alpha^{\prime \prime}(s) \beta^{\prime}(s)}{\left\|c_{2}^{\prime}\right\|^{3}} .
\end{aligned}
$$

are the differentiable functions. 
By the use of (4.7) with (2.2) we get the following result.

Remark 4.1. We have suppose that $c_{2}$ is not a straight line passing through the origin. In other case $M$ is a plane ([12]).

Lemma 4.1. Let $f=c_{1} \otimes c_{2}$ be tensor product immersion of a plane circle $c_{1}$ centered at 0 with any Euclidean planar curve $c_{2}(s)=(\alpha(s), \beta(s))$ then

$$
A_{N_{1}}=\left[\begin{array}{ll}
b(s) & 0 \\
0 & c(s)
\end{array}\right], A_{N_{2}}=\left[\begin{array}{ll}
0 & -b(s) \\
-b(s) & 0
\end{array}\right] .
$$

Thus by the use of (2.9) together with (2.14) and (2.15) we get the following result.

Proposition 4.1. Let $M$ a tensor product surface given with the surface patch (4.2). Then the Gaussian curvature $K$ coincides with the normal curvature $K_{N}$ of M. That is ;

$$
K=K_{N}=b(s)(c(s)-b(s)) .
$$

Corollary 4.1. Let $M$ a tensor product surface given with the surface patch (4.2). If $M$ has vanishing Gaussian curvature then $c_{2}$ is a logarithmic spiral given with the parametrization

$$
\alpha(s)=e^{\lambda s} \cos s, \beta(s)=e^{\lambda s} \sin s
$$

Proof. Suppose that $M$ has vanishing Gaussian curvature then substituting (4.8) into (4.10) we get

$$
\begin{gathered}
\left(\alpha^{\prime}(s) \beta(s)-\alpha(s) \beta^{\prime}(s)\right)\left\{\left(\alpha^{\prime}(s) \beta^{\prime \prime}(s)-\alpha^{\prime \prime}(s) \beta^{\prime}(s)\right)\left(\alpha^{2}(s)+\beta^{2}(s)\right)\right. \\
\left.+\left(\beta(s) \alpha^{\prime}(s)-\alpha(s) \beta^{\prime}(s)\right)\right\}=0 .
\end{gathered}
$$

So we have two possible cases;

$$
\beta(s) \alpha^{\prime}(s)-\alpha(s) \beta^{\prime}(s)=0
$$

or

$$
\left(\alpha^{\prime}(s) \beta^{\prime \prime}(s)-\alpha^{\prime \prime}(s) \beta^{\prime}(s)\right)\left(\alpha^{2}(s)+\beta^{2}(s)\right)+\left(\beta(s) \alpha^{\prime}(s)-\alpha(s) \beta^{\prime}(s)\right)=0 .
$$

For the first case $c_{2}$ is a straight line passing through the origin and $M$ becomes a plane. So this case does not occur. Further, from the differential equation in (4.14) we get $\alpha(s)=e^{\lambda s} \cos s, \beta(s)=e^{\lambda s} \sin s$. So, $c_{2}$ is a logarithmic spiral. For more details see also [11].

Theorem 4.1. Let $M$ a tensor product surface in $\mathbb{E}^{4}$ given with the surface patch (4.2). If $M$ is semi-parallel then it is a totally umbilical surface in $\mathbb{E}^{4}$.

Proof. Let $M$ be a tensor product surface in $\mathbb{E}^{4}$ given with the patch (4.2). Then by the use of (4.6) with (4.9) we get

$$
\begin{aligned}
& h_{11}^{1}=b(s), h_{12}^{1}=h_{21}^{1}=0, h_{22}^{1}=c(s), \\
& h_{11}^{2}=0, h_{12}^{2}=h_{21}^{2}=-b(s), h_{22}^{2}=0 .
\end{aligned}
$$

Using (2.5) with (4.15) we see that

$$
\begin{aligned}
h\left(X_{1}, X_{2}\right) & =-b(s) N_{2} \\
h\left(X_{1}, X_{1}\right)-h\left(X_{2}, X_{2}\right) & =(b(s)-c(s)) N_{1} .
\end{aligned}
$$


Further, substituting (4.15) and (4.16) into (3.2) and after some computation one can get

$$
\begin{aligned}
& \left(\bar{R}\left(X_{1}, X_{2}\right) \cdot h\right)\left(X_{1}, X_{1}\right)=-b(s)(b(s)(c(s)-b(s))+2 K) N_{2} \\
& \left(\bar{R}\left(X_{1}, X_{2}\right) \cdot h\right)\left(X_{1}, X_{2}\right)=\left(b^{2}(s)-K\right)(b(s)-c(s)) N_{1} \\
& \left(\bar{R}\left(X_{1}, X_{2}\right) \cdot h\right)\left(X_{2}, X_{2}\right)=-b(s)(c(s)(c(s)-b(s))-2 K) N_{2} .
\end{aligned}
$$

Suppose that, $M$ is semi-parallel then by definition $\left(\bar{R}\left(X_{1}, X_{2}\right) \cdot h\right)\left(X_{i}, X_{j}\right)=0,(1 \leq$ $i, j \leq 2)$ is satisfied. So, we get

$$
\begin{aligned}
b(s)(b(s)(c(s)-b(s))+2 K) & =0 \\
\left(b^{2}(s)-K\right)(b(s)-c(s)) & =0 \\
b(s)(c(s)(c(s)-b(s))-2 K) & =0 .
\end{aligned}
$$

Furthermore, substituting $K=b(s)(c(s)-b(s))$ into previous equation we obtain

$$
\begin{aligned}
b^{2}(s)(c(s)-b(s)) & =0, \\
b(s)(b(s)-c(s))(2 b(s)-c(s)) & =0 \\
b(s)(c(s)-b(s))(2 b(s)-c(s)) & =0 .
\end{aligned}
$$

Hence, two possible cases occur; either $b(s)=0$ or $b(s)=c(s)$. For the first case $c_{2}$ is a straight line passing through the origin and the surface $M$ becomes a plane. So we don't consider this case. Hence, $b(s)=c(s)$ which means that $R^{\perp}=0$ by (3.3) and (4.15). This is equivalent to say that $M$ has vanishing normal curvature $K_{N}$. So, $M$ is a totally umbilical surface in $\mathbb{E}^{4}$.

Example 4.1. If we take $c_{2}$ as a unit plane circle centered at 0 then the equation (4.12) is satisfied. In this case, surface patch becomes

$$
M: \quad f(t, s)=(\cos s \cos t, \sin s \cos t, \cos s \sin t, \sin s \sin t) .
$$

which is a Clifford torus in $\mathbb{E}^{4}$.

\section{REFERENCES}

[1] Arslan, K. and Murathan, C., Tensor Product Surfaces of Pseudo-Euclidean Planar Curves, Geometry and Topology of Submanifolds, VII, World Scientific, (1994), 71-75.

[2] Chen,B.Y., Geometry of Submanifolds, Dekker, New York, 1973.

[3] Chen, B.Y., Differential Geometry of Semiring of Immersions, I:General Theory, Bull. Inst. Math. Acad. Sinica, 21(1993),1-34.

[4] Decruyenaere, F., Dillen, F., Mihai, I., Verstraelen, L., Tensor Products of Spherical and Equivariant Immersions, Bull. Belg. Math. Soc. - Simon Stevin, 1(1994), 643-648.

[5] Decruyenaere, F., Dillen, F., Verstraelen, L., Vrancken, L., The Semiring of Immersions of Manifolds, Beitrage Algebra Geom. 34(1993), 209-215.

[6] Deprez, J., Semi-parallel Surfaces in Euclidean Space, J. Geom., 25(1985), 192-200.

[7] Deszcz, R., On Pseudosymmetric Spaces, Bull. Soc. Math. Belg., 44 ser. A, (1992), 1-34.

[8] Ferus, D., Symmetric Submanifolds of Euclidean Space, Math. Ann., 247(1980), 81-93.

[9] Guadalupe, I.V., Rodriguez, L., Normal Curvature of Surfaces in Space Forms, Pacific J. Math., 106(1983), 95-103.

[10] Lumiste, U., Classification of Two-codimensional Semi-symmetric Submanifolds. TRU Toimetised, 803(1988), 79-84.

[11] Mihai, I. and Rouxel, B., Tensor product surfaces of Euclidean plane curves, Results in Mathematics, 27 (1995), no. 3-4, 308-315.

[12] Mihai, I. and Rouxel, B., Tensor product surfaces of Euclidean Plane Curves, Geometry and Topology of Submanifolds, VII, World Scientific, (1994), 189-192.

[13] Mihai, I., Rosca, R., Verstraelen, L., Vrancken, L., Tensor Product Surfaces of Euclidean Planar Curves, Rend. Sem. Mat. Messina, 3(1994/1995), 173-184. 
[14] Mihai, I., Van de Woestyne, I., Verstraelen, L. and Walrave, J., Tensor Product Surfaces of Lorentzian Planar Curves, Bull. Inst. Math. Acad. Sinica, 23(1995), no.4, 357-363.

[15] Ozgur, C., Arslan, K., Murathan, C., On a Class of Surfaces in Euclidean Spaces, Commun. Fac. Sci. Univ. Ank. series A1, 51(2002), 47-54.

[16] Szabo, Z.I., Structure Theorems on Riemannian Spaces Satisfying R(X,Y) R $=0$. I. The LOCAL version, J. Differential Geometry, 17(1982), 531-582.

Department of Mathematics, Uludag University, Bursa -TURKey

E-mail address: bbulca@uludag.edu.tr

E-mail address: arslan@uludag.edu.tr 